\title{
Energy Expenditure During Incline Walking - Benefits of Integrating a Barometer into Activity Monitors
}

\author{
Armbruster Manuel ${ }^{1,2,{ }^{*}, \text { Anastasopoulou Panagiota }}{ }^{2}$, Altmann Stefan ${ }^{2}$, Ringhof Steffen ${ }^{2}$, \\ Neumann Rainer ${ }^{2}$, Haertel Sascha ${ }^{2}$, Woll Alexander ${ }^{2}$ \\ ${ }^{1}$ Department of Physiology and Biochemistry of Nutrition, Max Rubner-Institut, Karlsruhe, Germany \\ ${ }^{2}$ Institute of Sports and Sports Sciences, Karlsruhe Institute of Technology, Karlsruhe, Germany
}

\section{Email address:}

manuel.armbruster@mri.bund.de(A.Manuel)

${ }^{*}$ Corresponding author

\section{To cite this article:}

Armbruster Manuel, Anastasopoulou Panagiota, Altmann Stefan, Ringhof Steffen, Neumann Rainer, Haertel Sascha, Woll Alexander. Energy Expenditure During Incline Walking - Benefits of Integrating a Barometer into Activity Monitors. American Journal of Sports Science. Vol. 6, No. 2, 2018, pp. 47-54. doi: 10.11648/j.ajss.20180602.13

Received: February 15, 2018; Accepted: March 14, 2018; Published: April 4, 2018

\begin{abstract}
Objective: This study aimed to compare different methods to determine energy expenditure (EE) on incline walking. Approach: The methods tested were a conventional triaxial accelerometer (GT3X), a versatile system (SenseWear), both utilizing single regression models, and a device equipped with a triaxial accelerometer and an air pressure sensor (move II). Twenty-five healthy participants wore the activity monitors and a portable indirect calorimeter (IC) as reference while walking up- and downhill as well as up- and downstairs. The accuracy of the three devices for estimating EE was assessed based on Pearson correlation, ICC, and Bland-Altman analysis. Main results: For GT3X and SenseWear the ICCs showed a weak correlation (between 0.42 and 0.08 ) and for move II a strong correlation (between 0.97 and 0.84 ) between the prediction of energy cost and the output from IC, respectively. Overall, the differences absolute to the IC values were 11 to 35 (12 to 30 ) times higher for the GT3X (SenseWear) than for the move II devices. Significance: The study showed that a device equipped with an accelerometer and an air pressure sensor had higher accuracy in predicting EE during incline walking than a conventional accelerometer or a versatile system.
\end{abstract}

Keywords: Accelerometry, Validation, Indirect Calorimetry, Physical Activity

\section{Introduction}

Current levels of free-living physical activity, usually expressed as the amount of energy expended (EE) can be measured using a variety of methods. Those range from highly complex and expensive methods, such as the direct measurement of heat produced or the amount of oxygen inhaled and the carbon dioxide exhaled, to less unobtrusive methods with lower precision such as asking people to recall their past activities and fill in a questionnaire or a diary [1-3]. Accelerometry is the most commonly used objective method and has gained increasing importance in medical, sports and psychological science research in recent years [4-5]. This method enables estimation of different physical activity parameters from the type, frequency, duration and intensity of the activity.
Different validation studies using triaxial accelerometer (indirect calorimetry (IC) as reference method) have proven its validity to assess EE during walking [4-6], running [5], or other everyday activities [7-9]. The use of the aforementioned devices is restricted by the fact that they cannot assess the increase in energy expenditure during walking upstairs or uphill as the acceleration pattern remains unchanged under these conditions despite increased effort is required [4, 6, 10]. Different studies have shown the significant and partly heavy underestimation (30-50\%) of EE of walking upstairs or uphill $[5,7,11]$ as well as overestimation of walking downhill (up to $50 \%$ ) $[6,12]$. According to Hey et al., this non-linear coherence between acceleration data and energy expenditure leads to inadequate results [13].

A solution to this problem is the additional use of 
barometric sensors, which can determine the atmospheric pressure. In combination with an accelerometer, different movements can be classified more precisely and the accuracy of the EE calculation can be improved [14]. Wang et al. found an improvement ( $70 \%$ to $99 \%$ ) of the activity detection by integrating a barometer into the accelerometer. They analyzed walking (9 min), walking downstairs and walking upstairs (for $1.5 \mathrm{~min}$ ) in 13 young adults [7]. Furthermore, an obvious improvement in the accuracy of the EE calculation was also found by other researchers $[15,16]$.

The study of Anastasopoulou et al. using a conventional accelerometer and a device equipped with a triaxial accelerometer and a barometer confirmed these findings. Herein, walking up-/downhill was realized by use of a flat bridge (up to $5 \%$ ), but EEs were not calculated separately [17]. The weakness of the above-introduced studies is that they employed relatively slight inclines and, most important, the undifferentiated view of uphill and downhill movements using a triaxial accelerometer and a barometer. For a more accurate calculation, the separate consideration of uphill, downhill, upstairs, and downstairs walking is absolutely necessary to validate different EE estimation algorithms during incline walking and to generate revised and more precise algorithms.

Therefore, the purpose of this study was to compare different methods to determine the EE on inclines using the IC as reference method. The methods tested were a conventional accelerometer, a device equipped with a triaxial accelerometer and a barometer, and a versatile monitor which uses a combination of skin temperature, galvanic skin response, heat flux, and a biaxial accelerometer.

\section{Methods}

Twenty-five healthy participants volunteered to participate in the study. The study was approved from full ethics review by the institutional review board and was conducted in accordance with the Declaration of Helsinki [18]. All participants gave their written informed consent before participation. In order to have a sample that corresponds to the general adult population, participants with different ages, body characteristics, and physical condition of both sexes were chosen for the study. Exclusion criteria were chronic diseases, body impairments, and medication intake that could have a confounding effect to the implementation of the study. Descriptive data of the participants can be found in Table 1.

Table 1. Participant characteristics.

\begin{tabular}{|c|c|c|c|c|c|c|c|c|c|c|c|c|}
\hline & \multicolumn{4}{|c|}{$\operatorname{Men}(N=14)$} & \multicolumn{4}{|c|}{ Women $(N=11)$} & \multicolumn{4}{|c|}{$\operatorname{All}(\mathrm{N}=25)$} \\
\hline & Mean & SD & Min & Max & Mean & SD & Min & Max & Mean & SD & Min & Max \\
\hline Age [years] & 29.9 & 6.8 & 23.0 & 50.0 & 31.3 & 9.1 & 22.0 & 54.0 & 30.5 & 7.9 & 22.0 & 54.0 \\
\hline Height $[\mathrm{cm}]$ & 177.6 & 6.6 & 169.0 & 190.0 & 168.1 & 5.3 & 162.0 & 178.0 & 173.4 & 7.7 & 162.0 & 190.0 \\
\hline Mass [kg] & 75.2 & 7.7 & 60.0 & 88.0 & 61.9 & 7.0 & 53.0 & 76.0 & 69.4 & 9.9 & 53.0 & 88.0 \\
\hline BMI $\left[\mathrm{kg} / \mathrm{m}^{2}\right]$ & 23.8 & 2.2 & 19.4 & 27.4 & 21.9 & 1.6 & 19.7 & 24.9 & 23.0 & 2.2 & 19.4 & 27.4 \\
\hline
\end{tabular}

To compare the different activity monitors, the study design required the participants to wear all devices simultaneously, which were the move II (movisens $\mathrm{GmbH}$, Karlsruhe, Germany), the GT3X (ActiGraph Manufacturing Technology Inc., Pensacola, FL, USA), and the SenseWear (BodyMedia, Inc., Pittsburgh, PA, USA). The move II activity sensor consists of a triaxial acceleration sensor (adx1345, Analog Devices; range: $\pm 8 \mathrm{~g}$; sampling rate: $64 \mathrm{~Hz}$; resolution: 12 bit) and an air pressure sensor (BMP085, Bosch $\mathrm{GmbH}$; resolution: $0.03 \mathrm{hPa}$; sampling rate: $8 \mathrm{~Hz}$ ). The GT3X activity sensor consists of a triaxial acceleration sensor (adx1335, Analog Devices, Boston, USA; range: $\pm 3 \mathrm{~g}$; sampling rate: $30 \mathrm{~Hz}$; resolution: $12 \mathrm{bit}$ ). It is the most verified and widely used activity monitor and was therefore used as comparison for a conventional accelerometer [19].

The SenseWear armband device uses a combination of skin temperature, galvanic skin response, heat flux, and a biaxial accelerometer to estimate EE, physical activity duration and levels, and other parameters.

The activity sensors were attached to the participants' bodies according to the manufacturer's recommendations; that is right hip for move II and GT3X, and left upper arm on the top of the biceps for SenseWear, respectively. Additionally, a portable IC, MetaMax 3B (Cortex Biophysik, Leipzig, Germany), was worn as reference measure of the EE, and heart rate was monitored using a Polar Activity
Watch (Polar Electro Oy, Kempele, Finland). The portable IC consists of a face mask, a measurement and a battery module that were attached to the chest of the test participant using a harness. The device (breath-by-breath analysis) measures the oxygen consumption and carbon dioxide production to calculate the EE in one-second intervals. The validity and reliability of the MetaMax 3B is reported: both percentage errors and percentage technical error of measurements amount $<2 \%$ [20]. Prior to using, the system was turned on for at least $30 \mathrm{~min}$ and was then calibrated prior to every test according to the manufacturer's recommendations. Thereafter, all devices were initialized and synchronized using the respective software.

The data collection took place on a hill with both a walking path (approximately $0.75 \mathrm{~km}$ long and with $14 \%$ incline on average; incline varies from 5 to $20 \%$ ) and stairs (530 steps) that directly lead to the highest point of the hill, which is $103 \mathrm{~m}$ above the minimum point of the hill. Participants were asked to ascend/descend the stairs one step at a time, whereat no handrail was available. Activities were performed by all participants for both uphill/downhill and upstairs/downstairs walking at self-selected pace.

To assess the exact EE for each activity and to make sure that the participant had recovered from the physical effort needed for the previous activity, each activity was followed by a break. Since the body needs different recovery times for 
different activities and different intensities, the break was defined as time until the participant reached its resting heart rate or maximum $20 \%$ above its resting heart rate. The minimum time limit for the break was set to $5 \mathrm{~min}$.

The measured IC data were transferred to a laptop via wireless transmission and were analyzed using the associated software MetaSoft (Cortex Biophysik, Leipzig, Germany). The IC was used for both to collect the reference data for the $\mathrm{EE}$ and to set the start- and stop-markers for the different activities.

For the analysis of the move II data, the DataAnalyzer software was used. Based on the shared use of accelerometric and barometric data, new activity classes can be defined (walking uphill, upstairs, downhill or downstairs) and therefore separate algorithms were selected to calculate the EE. After entering the participant's physical characteristics (age, height, mass and sex) the software can estimate EE. The output sampling rate was set to $1 \mathrm{sec}$ [21].

For the analysis of the GT3X data, the software ActiLife 5.0 was used. By using the participant's mass, the activity energy expenditure (AEE) per second can be calculated. For the calculation of the AEE the "vector magnitude" algorithm was selected. Further information on the estimation of the AEE can be found in Sasaki et al [22].

For the analysis of the SenseWear data, the software SenseWear Professional 6.1 was used. To estimate EE, different participant characteristics must be entered in the software. Those include body height, mass, date of birth, gender, and whether the participant is a smoker or not. The output interval was pre-set to one minute.

Before evaluating and comparing the outputs of the different software, some pre-processing was necessary. First, the four outputs (output of the software plus reference) were synchronized based on the absolute time of the measurement and the markers from the IC were used to annotate the regions for the different activities. Since both the DataAnalyzer and the SenseWear Professional output the energy cost in total energy expenditure (TEE), whereas ActiLife provide the AEE, the Basal Metabolic Rate (BMR) was added to the output from ActiLife. BMR was estimated using the WHO formulas [23]. Finally, to compare the actual energy cost for each activity, steady state EE (based on IC) was identified, and the corresponding EE values were averaged and used for subsequent analyses using MS Excel (Microsoft Corporation, Redmond, USA).

All statistical analyses were performed using SPSS version 19.0 (SPSS Inc., Chicago, IL, USA). To investigate the agreement of the different estimation algorithms with respect to the criterion measure, a descriptive and a Bland-Altman analysis were performed. In the descriptive analysis, the bias with respect to criterion measure (both as absolute and as percent values) were computed. The Bland-Altman plots for each algorithm and each activity were calculated and the measurement errors of both devices were plotted against their mean. A zero bias represents that no difference exists between actual and estimated EE, a negative bias (IC EE predicted $\mathrm{EE}$ ) indicates an underestimation of $\mathrm{EE}$ by the estimation algorithm, whereas a positive bias corresponds to an overestimation of EE by the estimation algorithm. The $95 \%$ limits of agreement were calculated as mean $\pm 1.96 * \mathrm{SD}$. The smaller the range between these two limits the more accurate the algorithm is. Additionally, Pearson and intraclass correlations for absolute agreement (ICC) were calculated. P-values $<0.05$ were considered to indicate statistical significance. All data followed a normal distribution.

\section{Results}

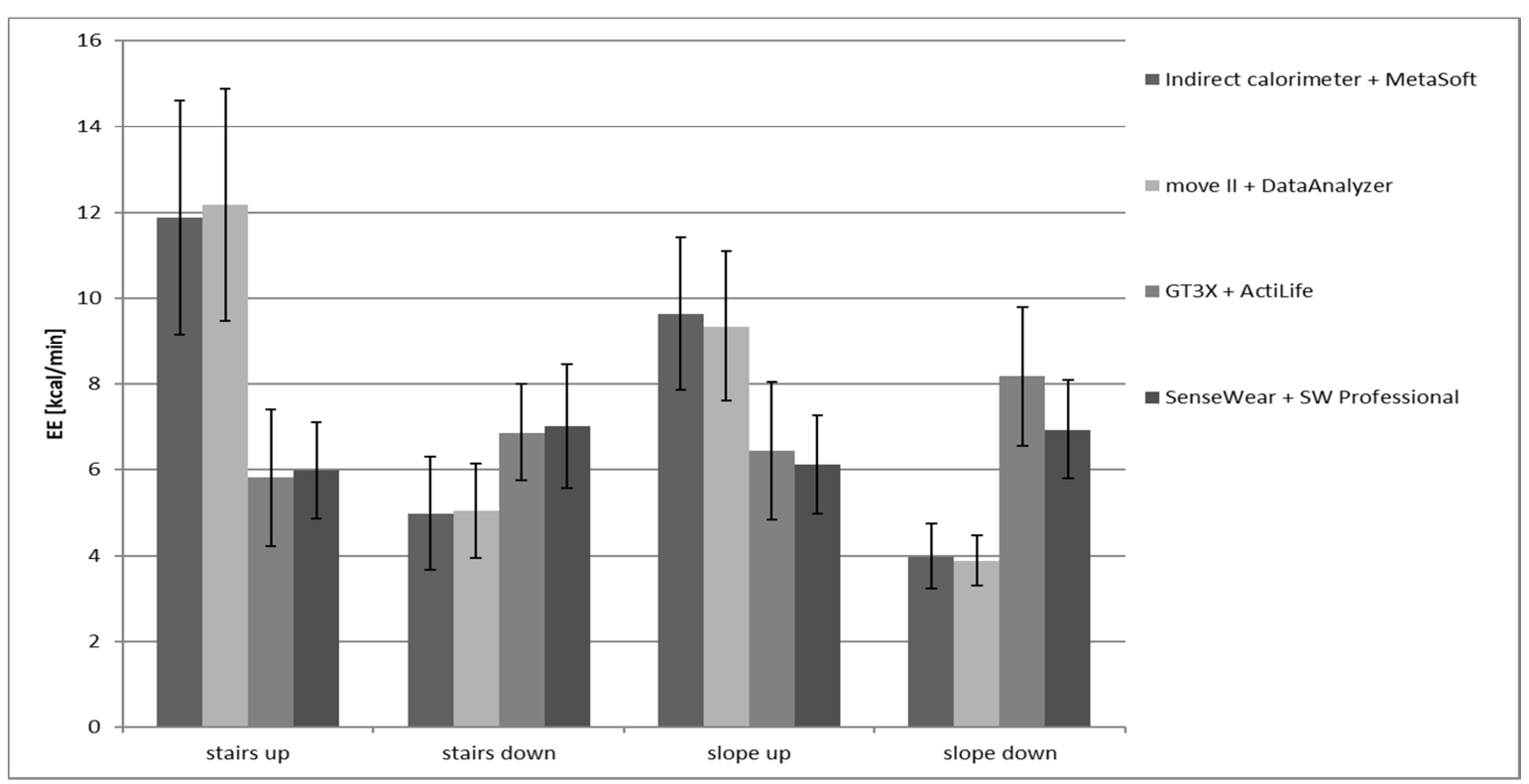

Figure 1. Mean EE for the 3 devices and the IC during the different activities.

Figure 1 shows the mean EE for each device and the IC for the different activities. The results for the move II follow the 
ones of the IC; the results for the other two devices differ with respect to the IC but have similar results when compared to one another.

Table 2. Comparison of EE in kcal/min for the different activities [mean (SD)].

\begin{tabular}{|c|c|c|c|c|c|c|c|}
\hline $\begin{array}{l}\text { Activity Time[sec] } \\
\text { Averaged velocity }[\mathrm{m} / \mathrm{sec}]\end{array}$ & IC [kcal/min] & Device & $\begin{array}{l}\text { measure } \\
{[\mathrm{kcal} / \mathrm{min}]}\end{array}$ & bias [kcal/min] & bias [\%] & $\mathbf{r}^{1}$ & $\mathrm{ICC}^{2}$ \\
\hline Walking upstairs & \multirow{3}{*}{$11.89(2.73)$} & move II & $12.18(2.70)$ & $0.29(0.56)$ & $2.7(4.6)$ & $0.98 *$ & $0.97 *$ \\
\hline $458(66)$ & & GT3X & $5.82(1.59)$ & $-6.07(1.85)$ & $-50.7(8.2)$ & $0.76^{*}$ & $0.14 *$ \\
\hline $1.16(0.17)$ & & SenseWear & $5.98(1.12)$ & $-5.91(2.06)$ & $-48.5(9.2)$ & $0.72 *$ & $0.11^{*}$ \\
\hline Walking downstairs & \multirow{3}{*}{$4.97(1.32)$} & move II & $5.04(1.10)$ & $0.07(0.44)$ & $3.0(9.5)$ & $0.95 *$ & $0.94 *$ \\
\hline $362(64)$ & & GT3X & $6.87(1.13)$ & $1.91(1.16)$ & $44.1(28.5)$ & $0.58^{*}$ & $0.27 *$ \\
\hline $1.47(0.29)$ & & SenseWear & $7.01(1.45)$ & $2.05(0.76)$ & $44.8(22.5)$ & $0.86^{*}$ & $0.42 *$ \\
\hline Walking uphill & \multirow{3}{*}{$9.63(1.77)$} & move II & $9.34(1.74)$ & $-0.29(0.45)$ & $-2.9(4.6)$ & $0.97 *$ & $0.96 *$ \\
\hline $602(62)$ & & GT3X & $6.44(1.61)$ & $-3.19(1.47)$ & $-32.8(12.1)$ & $0.63 *$ & $0.23 *$ \\
\hline $1.23(0.12)$ & & SenseWear & $6.12(1.15)$ & $-3.51(1.16)$ & $-35.9(8.6)$ & $0.76^{*}$ & $0.19 *$ \\
\hline Walking downhill & \multirow{3}{*}{$3.98(0.77)$} & move II & $3.87(0.59)$ & $-0.12(0.38)$ & $-1.9(8.3)$ & $0.88^{*}$ & $0.84 *$ \\
\hline $491(34)$ & & GT3X & $8.18(1.61)$ & $4.20(1.24)$ & $107.2(27.8)$ & $0.66^{*}$ & $0.08^{*}$ \\
\hline $1.51(0.11)$ & & SenseWear & $6.93(1.15)$ & $2.94(0.60)$ & $75.7(17.2)$ & $0.84 *$ & $0.14 *$ \\
\hline
\end{tabular}

1: Pearson correlation coefficient between the device (move II, GT3X, SenseWear) and the reference IC.

${ }^{2}:$ Intra class correlation coefficient (absolute agreement) for the device and the reference IC.

*: statistical significant at.05

Table 2 shows the descriptive statistics for EE as measured from the IC, the move II, the GT3X, and the SenseWear as well as the mean and percent differences between the criterion measure and the three monitoring devices for each activity, respectively. Furthermore, the Pearson correlation and the agreement between the predicted values and the IC (ICC) are presented.

stairs up

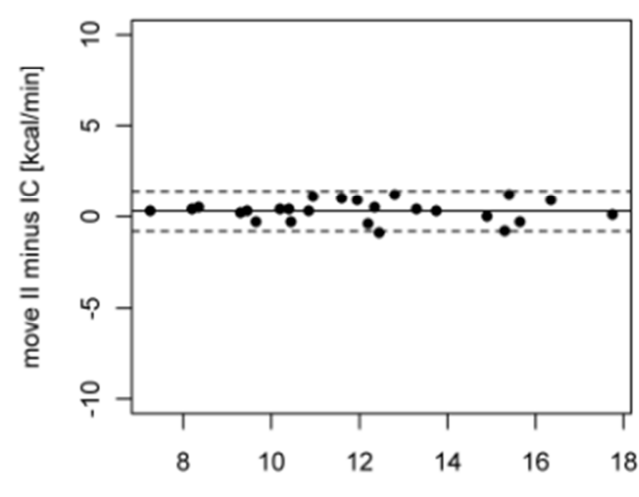

Mean of IC and move II [kcal/min]

slope up

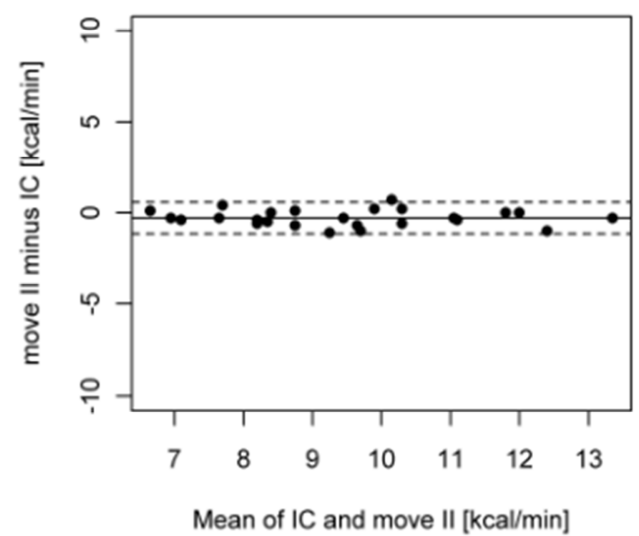

stairs down

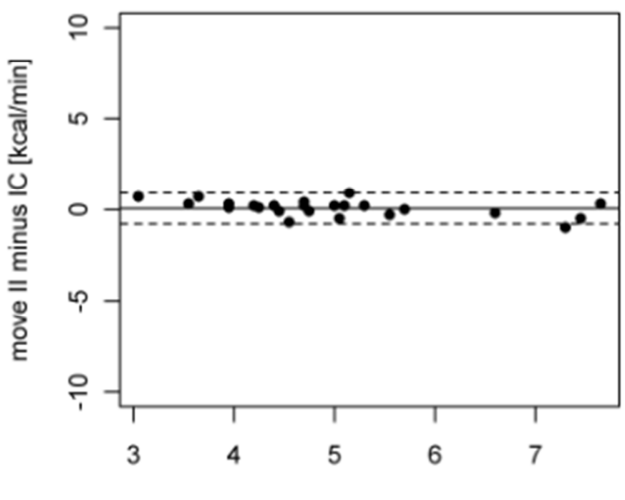

Mean of IC and move II [kcal/min]

slope down

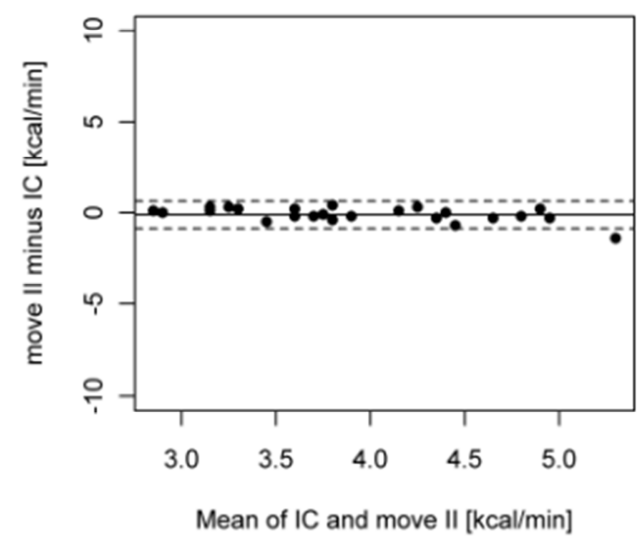

Figure 2. Bland-Altman plots for move II. The solid lines represent the mean bias between actual and calculated EE, and the dashed lines represent the limits of agreement ( $\pm 1.96 \mathrm{SDs}$ ). 
stairs up

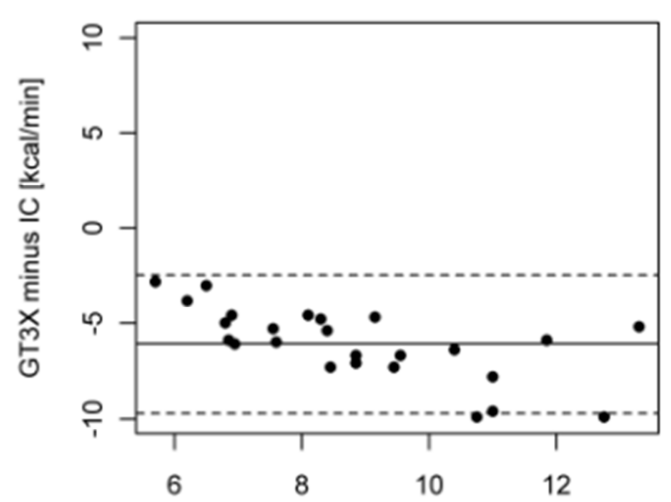

Mean of IC and GT3X [kcal/min]

slope up

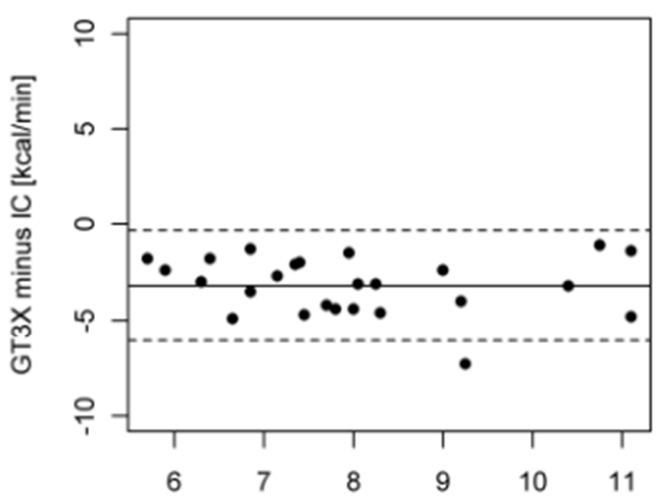

Mean of IC and GT3X [kcal/min] stairs down

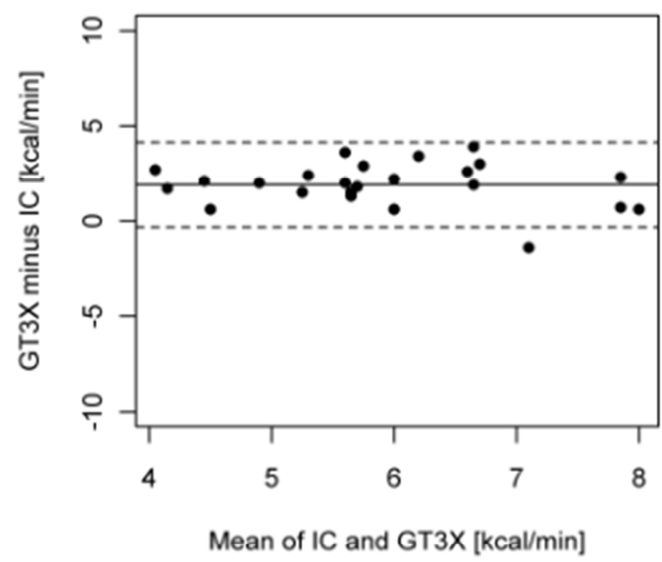

slope down

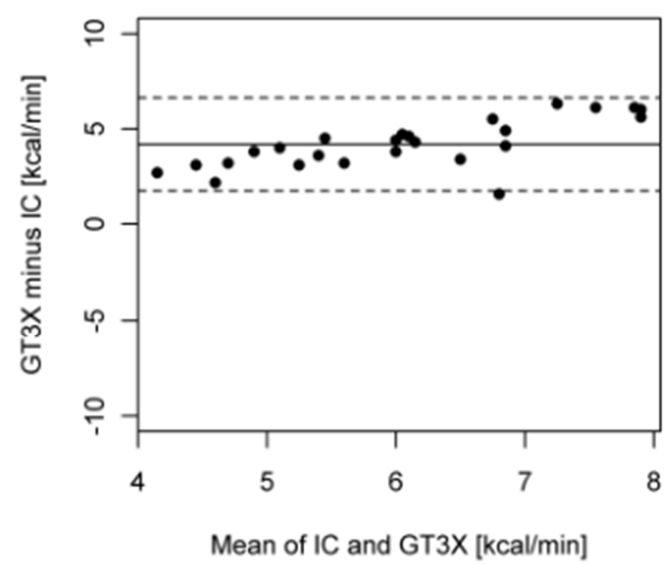

Figure 3. Bland-Altman plots for GT3X. The solid lines represent the mean bias between actual and calculated EE, and the dashed lines represent the limits of agreement $( \pm 1.96 \mathrm{SDs})$

stairs up

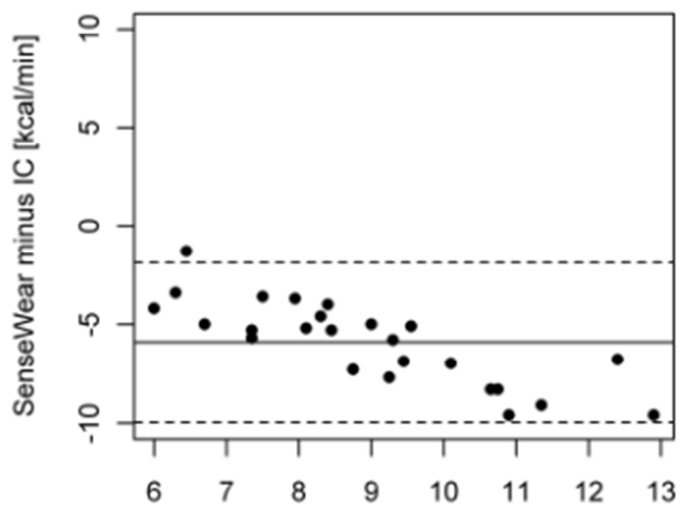

Mean of IC and SenseWear [kcal/min] stairs down

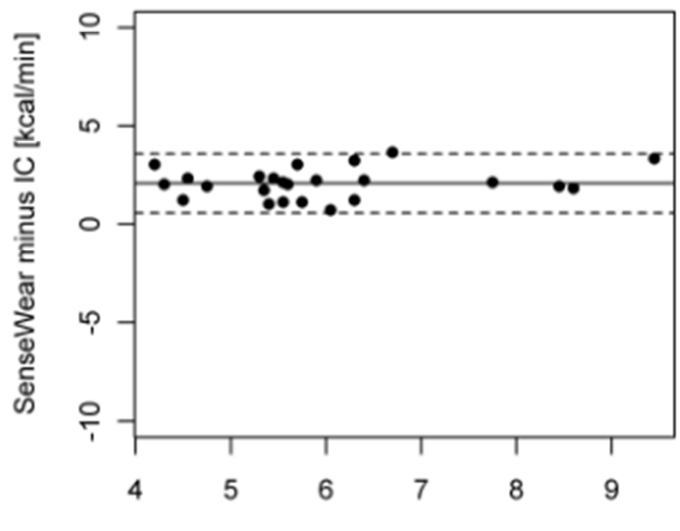

Mean of IC and SenseWear [kcal/min] 

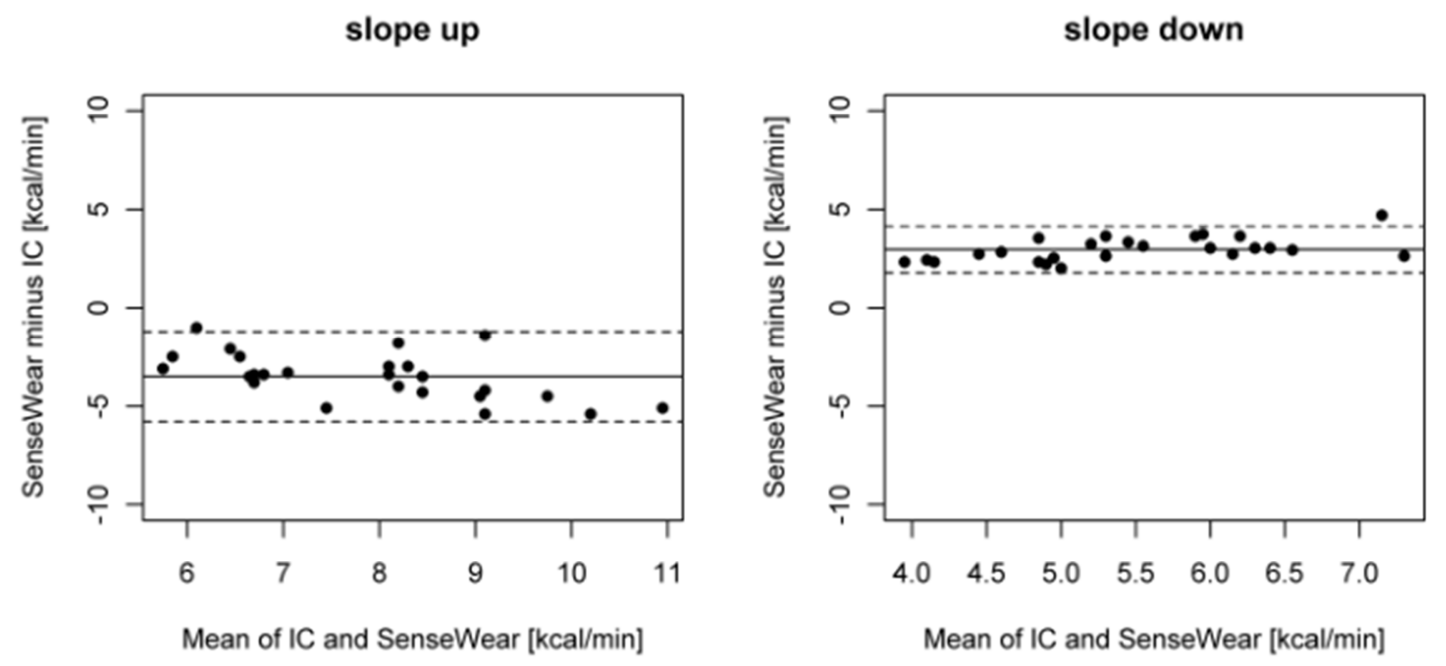

Figure 4. Bland-Altman plots for SenseWear. The solid lines represent the mean bias between actual and calculated EE, and the dashed lines represent the limits of agreement $( \pm 1.96 \mathrm{SDs})$.

The Bland-Altman plots for the GT3X, the move II, and the SenseWear are shown in Figures 2-4. Overall, the differences absolute to the IC values were 11 to 35 and 12 to 30 times higher for the GT3X and SenseWear than for the move II devices, respectively.

\section{Discussion}

The aim of this study was to compare different methods for estimating EE on inclines against the IC. Two devices (GT3X, SenseWear) use the commonly used single regression model for estimating EE, and the other device (move II) first detects the type of the activity and then uses the respective regression model for estimating EE. Furthermore, the move II uses data of acceleration and pressure for estimating EE. The latter approach was found to be more accurate for estimating EE on inclines, which is in agreement with previous studies [7, 16, 17].

In general, both the GT3X and the SenseWear underestimated EE while walking upstairs and uphill, and overestimated EE while walking downstairs and downhill. Those findings are in line with previous results $[6,11,12]$ where solely accelerometry was proven insufficient for estimating EE while walking on inclines. Additionally, both devices showed similar results for all investigated activities, thus, leading to the suggestion that a versatile monitor, which uses a combination of skin temperature, galvanic skin response, heat flux, and a biaxial accelerometer provides no significant improvement in the EE estimation - at least not for the tested activities.

The largest errors (107\%) in estimating EE were observed during walking downhill with the GT3X $(76 \%$ with SenseWear). With the exception of one participant, the estimated EE by GT3X and SenseWear of any participant during walking uphill/upstairs (downhill/downstairs) was underestimated (overestimated). For GT3X and SenseWear, the ICCs showed a weak correlation $(0.42$ to 0.08$)$ between the prediction of energy cost and the output of the indirect calorimeter.

The above-discussed is an existing limitation of the acceleration-based activity monitors [6]. They are not able to assess the increase in energy cost of walking upstairs or uphill because the acceleration pattern remains very similar to that for normal walking despite an increased effort is required to elevate the body's center of mass. As a result, they tend to underestimate the EE for these activities. In contrast, for descending stairs or walking downhill the acceleration magnitude is greater although the effort remains almost the same. This results in an overestimation of the EE. This may be due to the fact that there is no linear relationship between the acceleration and the EE across all different types of activities, since different types of activities include the use of different muscles. Because of the even surface by walking downstairs, the increase of the acceleration data was only slight and, in turn, also the overestimation of the EE. In contrast, the overestimation during walking downhill could be substantially higher due to the enormous increase of the acceleration data (sloping surface). The increased values measured lead to a misinterpretation of the data and, therefore, to an incorrect estimation of EE.

The move II was more accurate in estimating EE for walking uphill/upstairs and downhill/downstairs. The ICCs showed a strong correlation (between 0.97 and 0.84 ) between the prediction of energy cost and the output of the indirect calorimeter. This finding is supported by the results of Bland-Altman analysis. The relative bias was very slight and lay between $-3 \%$ and $3 \%$. No clear trend to an over- or underestimation of the EE in any of the activities could be recognized. By comparing the results with previous studies using devices without a barometer, an increased accuracy for estimating EE was found [7, 15, 16].

Although we recruited participants with different body characteristics, the final test sample included only young to middle aged participants ( 22 to 54 years) and, therefore, the results cannot be generalized for other population groups (e.g. elderly people, children, obese). 
Moreover, the BMR was calculated using the WHO formulas instead of measuring it using the indirect calorimeter [23]. The formula shows a high validity as well as a good coincidence with IC [24]. Besides, the BMR plays only a small role in relation to the EE during the activities studied. The error rate should therefore be very low.

It was not possible to unambiguously clarify whether the minimum break of 5 minutes between the different activities was sufficient. Different researchers found that the oxygen consumption after an intense exercise will return from elevated levels of expenditure to normal values within a few minutes. Anyway, nobody could indicate the exact number of minutes required [25]. Due to the fact that the actual heart rate in relation to the resting heart rate was considered, the length of the break should be enough to recover [25]. Moreover, as we used the same schedule for every participant there should be no falsifying influence. A further limitation of the current study is that no different inclinations in terms of steepness where investigated. However, the inclines vary from $5 \%$ to $20 \%$ and therefore reflect a wide range of inclinations occurring during walking activities.

\section{Conclusion}

We conclude that EE predictions from single use of accelerometry should be considered with caution when investigating inclined walking or walking on uneven terrain. Using more comprehensive EE prediction models depending on the type of activity being performed and expanding accelerometry with air pressure data (barometers) improve the EE estimation. The technology of the move II not only utilizes activity-based prediction models but also comprises a barometer, and hence accounts for the differences in center of mass elevation. This approach results in smaller errors in estimating EE for walking up- and downstairs (up- and downhill) compared to those of the GT3X and the SenseWear device. Consequently, for activities involving elevation gain or loss, the additional use of barometer data for estimating EE seems to be appropriate.

\section{References}

[1] Sirard J. R., Pate R. R. Physical activity assessment in children and adolescents. Sports medicine (Auckland, N. Z.). 2001; 31(6): 439-454.

[2] Falck R. S., McDonald S. M., Beets M. W., Brazendale K., Liu-Ambrose T. Measurement of physical activity in older adult interventions: a systematic review. British journal of sports medicine. 2016; 50(8): 464-470.

[3] Sun F., Norman I. J., While A. E. Physical activity in older people: a systematic review. BMC public health. 2013; 13: 449.

[4] Perrin O., Terrier P., Ladetto Q., Merminod B., Schutz Y. Improvement of walking speed prediction by accelerometry and altimetry, validated by satellite positioning. Medical \& biological engineering \& computing. 2000; 38(2): 164-168.
[5] Campbell K. L., Crocker P. R., McKenzie D. C. Field evaluation of energy expenditure in women using Tritrac accelerometers. Medicine and science in sports and exercise. 2002; 34(10): 1667-1674.

[6] Terrier P., Aminian K., Schutz Y. Can accelerometry accurately predict the energy cost of uphill/downhill walking? Ergonomics. 2001; 44(1): 48-62.

[7] Wang J., Redmond S. J., Voleno M., Narayanan M. R., Wang N., Cerutti S., Lovell N. H. Energy expenditure estimation during normal ambulation using triaxial accelerometry and barometric pressure. Physiological measurement. 2012; 33(11): 1811-1830.

[8] Jeran S., Steinbrecher A., Pischon T. Prediction of activityrelated energy expenditure using accelerometer-derived physical activity under free-living conditions: a systematic review. International journal of obesity. 2016; 40(8): 11871197.

[9] Daniel C. R., Battistella L. R. Validation of accelerometry for measuring energy expenditure: a systematic review. Acta Fisiatr. 2014; 21(2): 87-92.

[10] Vernillo G., Savoldelli A., Pellegrini B., Schena F. Validity of the SenseWear Armband to assess energy expenditure in graded walking. Journal of physical activity \& health. 2015; 12(2): 178-183.

[11] Chen K. Y., Sun M. Improving energy expenditure estimation by using a triaxial accelerometer. Journal of applied physiology (Bethesda, Md.: 1985). 1997; 83(6): 2112-2122.

[12] Crouter S. E., Churilla J. R., Bassett D. R., Jr. Estimating energy expenditure using accelerometers. European journal of applied physiology. 2006; 98(6): 601-612.

[13] Hey S., Anastasopoulou P., Haaren B. Erfassung körperlicher Aktivität mittels Akzelerometrie - Möglichkeiten und Grenzen aus technischer Sicht. $B$ \& G. 2014; 30(2): 73-78.

[14] Yang C. C., Hsu Y. L. A review of accelerometry-based wearable motion detectors for physical activity monitoring. Sensors (Basel, Switzerland). 2010; 10(8): 7772-7788.

[15] Yamazaki T., Gen-No H., Kamijo Y., Okazaki K., Masuki S., Nose $\mathrm{H}$. A new device to estimate VO2 during incline walking by accelerometry and barometry. Medicine and science in sports and exercise. 2009; 41(12): 2213-2219.

[16] Duncan G. E., Lester J., Migotsky S., Higgins L., Borriello G. Measuring slope to improve energy expenditure estimates during field-based activities. Applied physiology, nutrition, and metabolism = Physiologie appliquee, nutrition et metabolisme. 2013; 38(3): 352-356.

[17] Anastasopoulou P., Tubic M., Schmidt S., Neumann R., Woll A., Hartel S. Validation and comparison of two methods to assess human energy expenditure during free-living activities. PloS one. 2014; 9(2): e90606.

[18] World Medical Association Declaration of Helsinki: ethical principles for medical research involving human subjects. JAMA: the journal of the American Medical Association. 2013; 310(20): 2191-2194.

[19] Hills A. P., Mokhtar N., Byrne N. M. Assessment of physical activity and energy expenditure: an overview of objective measures. Frontiers in nutrition. 2014; 1: 5. 
[20] Macfarlane D. J., Wong P. Validity, reliability and stability of the portable Cortex Metamax 3B gas analysis system. European journal of applied physiology. 2012; 112(7): 25392547.

[21] Anastasopoulou P., Tansella M., Stumpp J., Shammas L., Hey S. Classification of human physical activity and energy expenditure estimation by accelerometry and barometry. Conference proceedings.... Annual International Conference of the IEEE Engineering in Medicine and Biology Society. IEEE Engineering in Medicine and Biology Society. Conference. 2012; 2012: 6451-6454.

[22] Sasaki J. E., John D., Freedson P. S. Validation and comparison of ActiGraph activity monitors. Journal of science and medicine in sport / Sports Medicine Australia. 2011; 14(5): 411-416.

[23] WHO. Energy and protein requirements. Report of a joint FAO/WHO/UNU Expert Consultation. World Health Organization technical report series. 1985; 724: 1-206.

[24] Henry C. J. Basal metabolic rate studies in humans: measurement and development of new equations. Public health nutrition. 2005; 8(7a): 1133-1152.

[25] Hollmann W., Strüder H. K., Tagarakis C. V. Spiroergometrie: Kardiopulmonale Leistungsdiagnostik des Gesunden und Kranken, Stuttgart, Schattauer; 2006. 